ISSN 0103-5150

Fisioter. Mov., Curitiba, v. 25, n. 1, p. 73-81, jan./mar. 2012 Licenciado sob uma Licença Creative Commons

\title{
Qualidade de vida e funcionalidade em adolescentes com escoliose idiopática: estudo piloto
}

\author{
Quality of life and functional in adolescents with \\ idiopathic scoliosis: pilot study
}

\author{
Laís Fernandes $^{[a]}$, Milena Carlos Vidotto Crescentini ${ }^{[b]}$, Patrícia Rios Poletto ${ }^{[c]}$, Alberto Ofenhejm \\ Gotfryd $^{[\mathrm{d}]}$, Liu Chiao Yi ${ }^{[e]}$ \\ [a] Graduanda do curso de Fisioterapia da Universidade Federal de São Paulo (UNIFESP), São José dos Campos, SP - Brasil, \\ e-mail: lais_fernandess@hotmail.com \\ [b] Doutora, docente do curso de Fisioterapia da Universidade Federal de São Paulo (UNIFESP), Santos, SP - Brasil, e-mail: \\ milenavidotto@hotmail.com \\ [c] Doutora, docente do curso de Fisioterapia da Universidade Federal de São Paulo (UNIFESP), Santos, SP - Brasil, e-mail: \\ patricia.poletto@gmail.com \\ [d] Mestre, médico chefe do Ambulatório de Coluna Vertebral da Irmandade da Santa Casa da Misericórdia de Santos, Santos, \\ SP - Brasil, e-mail: albertocoluna@yahoo.com.br \\ [e] Doutora, docente do curso de Fisioterapia da Universidade Federal de São Paulo (UNIFESP), Santos, SP - Brasil, e-mail: \\ liuchiaoyi@yahoo.com.br
}

\section{Resumo}

Introdução: A escoliose idiopática do adolescente (EIA) é uma deformidade tridimensional, na qual sua progressão pode levar à diminuição da qualidade de vida (QDV). Objetivos: Verificar o melhor questionário, SF-36 ou SRS-22, para avaliar a QDV na EIA. Secundariamente, avaliar a correlação entre o grau do ângulo de Cobb com a escala visual analógica (EVA) e verificar qual o melhor questionário, Oswestry ou Roland Morris, para avaliar a funcionalidade na EIA. Materiais e métodos: Participaram 19 indivíduos com EIA, idade entre 11 a 18 anos e ângulo de Cobb acima de $25^{\circ}$. Foram utilizados os questionários SF-36 e SRS-22, Oswestry e Roland Morris e a EVA. Os questionários e a escala foram correlacionados com os ângulos de Cobb torácico proximal, torácico principal e lombar. Resultados: A população avaliada apresentou média de idade de 14,5 (3,1), peso $51 \mathrm{~kg}(7,9)$, altura 1,61 (0,08) e IMC 18,75 (2,7). Os coeficientes de correlação de Pearson entre o questionário Oswestry e os ângulos de Cobb torácico proximal, torácico principal e lombar foram, respectivamente: $r=0,58, r=0,77$ e $r=0,58$. Ao correlacionar o questionário Roland Morris com os 
ângulos de Cobb torácico proximal e lombar, os coeficientes foram, respectivamente: $r=0,72$ e $r=0,52$. Ao correlacionar o Cobb lombar com o EVA, foi encontrado $r=0,55$. Conclusão: Foi constatada forte correlação entre o questionário Oswestry com o ângulo de Cobb torácico principal e entre o questionário Roland Morris com o ângulo de Cobb torácico principal.

Palavras-chave: Escoliose. Adolescente. Qualidade de vida. Questionário.

\begin{abstract}
Introduction: Adolescent with idiopathic scoliosis (AIS) presents a three-dimensional deformity in which progression can lead to decreased quality of life (QOL). Objectives: Determine the best questionnaire, SF-36 or SRS-22 to evaluate QOL in EIA. Secondarily, investigate the correlation between the degree of Cobb angle with visual analogue scale (VAS), and determine the best questionnaire, Oswestry or Roland Morris to evaluate the function. Materials and methods: 19 subjects with EIA, aged 11 to 18, both gender, and Cobb angle above 25 Cobb degrees. We used the SF-36 and SRS-22 questionnaires to evaluate QOL, Oswestry and Roland Morris to evaluate the function and correlate Cobb degree and VAS. The questionnaires and scale were correlated with proximal thoracic, main thoracic and lumbar Cobb angles. Results: The population studied had a mean age of 14.5 (3.1), weight $51 \mathrm{~kg}$ (7.9), height $1.61 \mathrm{~m}$ (0.08), BMI 18.75 (2.7). The Pearson correlation coefficients between the Oswestry questionnaire and the Cobb angles proximal thoracic, main thoracic and lumbar spine were, respectively: $r=0.58, r=0.77$ and $r=0.58$. By correlating the Roland Morris questionnaire with Cobb angles of proximal thoracic and lumbar spine were, respectively: $r=0.72$ and $r=0.52$. By correlating with lumbar Cobb EVA was found $r=0.55$. Conclusion: We found a strong correlation between the Oswestry questionnaire with the main thoracic Cobb angle and between the Roland Morris questionnaire with the main thoracic Cobb angle.
\end{abstract}

Keywords: Adolescent. Scoliosis. Quality of life. Questionnaire.

\section{Introdução}

Escoliose idiopática do adolescente (EIA) é uma deformidade tridimensional que apresenta na coluna vertebral uma ou mais curvas no plano frontal, rotação axial das vértebras no plano transverso e alteração do alinhamento no plano sagital $(1,2)$. Segundo a Scoliosis Research Society (3), o desvio da coluna deve ser superior a 10 graus, calculado pelo Método de Cobb, sendo que curvaturas abaixo desse valor são consideradas variações da normalidade $(4,5)$.

A denominação de EIA é dada aos indivíduos com idade entre 11 a 18 anos, com prevalência de 3\%-5\% nessa faixa etária e sem causa aparente $(5$, 6). Aproximadamente $85 \%$ das escolioses em adolescentes são de origem idiopática e sua incidência e evolução são maiores no gênero feminino $(6,7)$. A progressão da EIA pode levar a alterações cardiorrespiratórias e dor lombar com consequente redução da qualidade de vida nos adolescentes, sendo mostrado em alguns estudos que esses indivíduos relatam se sentirem menos saudáveis e restritos em atividades físicas e sociais (8).

A QDV abrange os domínios da saúde, habilidades funcionais nas atividades do dia a dia, aspectos psicológicos e sociais que serão afetados significativamente com o aumento da EIA (9). O comprometimento mais citado na EIA e que poderá interferir na qualidade de vida é a alteração da imagem corporal causada pela deformidade tridimensional da coluna vertebral acometendo a simetria do tronco e segmentos satélites (8).

$\mathrm{Na}$ prática clínica, foi observado que pacientes com EIA apresentam algia em coluna vertebral que pode interferir nas atividades funcionais. Questiona-se qual o melhor instrumento para a avaliação de atividades funcionais, que melhor correlaciona com os principais desvios laterais da coluna vertebral, possibilitando melhor acompanhamento na evolução e no tratamento desses indivíduos.

Muitas correlações têm sido feitas para identificar quais aspectos estão associados com o declínio na qualidade de vida na EIA. As mais citadas 
na literatura são o tipo de tratamento, gênero e a deformidade escoliótica mensurada pelo método de Cobb (7). Muitos questionários avaliam a QDV, sendo o SF-36 o mais utilizado (9). No entanto, este é colocado por Martinez (10) como genérico, pois seus conceitos não são específicos para uma determinada idade, doença ou grupo de tratamento. Outro questionário é o Scoliosis Research Society versão 22 (SRS-22), que é específico para avaliar a qualidade de vida em indivíduos com escoliose idiopática, havendo uma versão modificada do SRS-24 que, apesar de sua brevidade e interpretação simples dos resultados, teve algumas falhas metodológicas $(11,12)$. 0 questionário SF-36 é o principal questionário usado para verificar a qualidade de vida independente das características da população (10).

Visto que as alterações musculoesqueléticas nos indivíduos com EIA podem levar a uma diminuição na qualidade de vida, constatou-se a importância de verificar o melhor questionário, genérico ou específico, para avaliar a qualidade de vida nessa população.

\section{Objetivo principal}

Verificar o melhor questionário, SF-36 (genérico) ou SRS-22 (específico), para avaliar a qualidade de vida em indivíduos com EIA.

\section{Objetivos secundários}

- Verificar o melhor questionário, Oswestry ou Roland Morris, para avaliar a funcionalidade em indivíduos com EIA;

- verificar a correlação entre os ângulos de Cobb com a dor.

\section{Materiais e métodos}

Tipo de estudo: observacional transversal

Foram avaliados 25 pacientes provenientes do Departamento de Ortopedia e Traumatologia da Irmandade da Santa Casa da Misericórdia de Santos, com diagnóstico de EIA, que apresentam o ângulo de Cobb torácico principal superior a $25^{\circ}$, de ambos os gêneros, na faixa etária de 11 a 18 anos de idade.
Foram incluídos no estudo pacientes que não estejam realizando ou tenham realizado tratamento fisioterapêutico ou cirúrgico. Foram excluídos indivíduos com história prévia ou atual de doenças cardiorrespiratórias, doenças musculoesqueléticas ou neuromusculares sistêmicas.

Atenderam aos critérios estipulados 19 pacientes. As avaliações foram realizadas no Laboratório de Estudos da Motricidade Humana da Universidade Federal de São Paulo (Unifesp) - Câmpus Baixada Santista. O estudo foi aprovado pelo Comitê de Ética e Pesquisa (CEP): 1.261/10. Os voluntários do estudo assinaram o Termo de Consentimento Livre e Esclarecido.

Todos os participantes foram submetidos à anamnese para identificação dos dados pessoais, história da moléstia atual e pregressa, e responderam aos questionários elencados na sequência.

Questionário de Qualidade de Vida (SF-36)

Trata-se de um questionário multidimensional, validado para a língua portuguesa, de avaliação da saúde, formado por um total de 36 itens, subdivididos em oito domínios: 1) capacidade funcional (dez itens); 2) aspectos físicos (quatro itens); 3) dor (dois itens); 4) estado geral de saúde (cinco itens); 5) vitalidade (quatro itens); 6) aspectos sociais (dois itens); 7) aspectos emocionais (três itens); 8) saúde mental (cinco itens); e ainda uma questão que compara as condições de saúde atual e a do ano anterior. Uma pontuação de $0 \mathrm{a}$ 100 é dada para cada domínio e quanto maior a pontuação melhor o estado de saúde $(13,14)$.

Scoliosis Research Society - versão 22 (SRS- 22)

É um questionário usado em pacientes com escoliose idiopática, contendo 22 questões que abrangem cinco domínios: 1) função/atividade; 2) autoimagem/ aparência; 3) saúde mental; 4) dor; 5) satisfação com o tratamento. A pontuação é fornecida para cada item expresso em uma escala de um a cinco pontos, sendo cinco a melhor condição de saúde $(15,16)$.

\section{Escala visual analógica de dor (EVA)}

Consiste em uma escala de 10 centímetros, graduada de 0 a 10.0 paciente fornece uma nota para 
sua dor de forma global nos últimos sete dias, que pode variar entre os dois extremos $(0=$ sem dor e $10=$ dor extrema). Quanto maior a pontuação, pior a dor (17).

\section{Roland Morris}

Trata-se de um questionário que avalia a funcionalidade de pacientes que sofrem dor na coluna lombar. É composto por 24 questões que verificam a deficiência como resultado da dor na coluna lombar. As questões são frases que as pessoas têm utilizado para se descreverem quando sentem dores nas costas. As respostas são de sim ou não para cada questão, e o resultado é dado pelo número de itens marcados, de um mínimo de 0 a um máximo de 24 pontos. Quanto maior a pontuação, maior é a incapacidade do indivíduo (18).

\section{Oswestry}

Questionário feito para a avaliação da dor lombar. É composto por dez questões com seis alternativas de resposta, sendo a primeira relacionada com a intensidade de dor e as demais descrevendo atividades da vida diária que podem ser prejudicadas e/ ou incapacitadas pela dor na coluna lombar. A pontuação é de 0 - sem nenhuma invalidez - a 100, que é a máxima invalidez (19).

Os valores angulares dos desvios laterais da coluna vertebral (torácico proximal, principal e lombar) foram medidos pelo método de Cobb e avaliados pelo médico responsável pelo Ambulatório de Coluna Vertebral da Irmandade da Santa Casa da Misericórdia de Santos.

\section{Medida do ângulo de Cobb}

A mensuração da curva escoliótica pelo método de Cobb foi realizada utilizando-se radiografias panorâmicas da coluna vertebral nas incidências póstero-anteriores e laterais. 0 primeiro passo consistiu em identificar as vértebras terminais. Uma linha foi traçada na extremidade superior da vértebra terminal cranial, ao longo da placa terminal; outra linha foi traçada perpendicularmente com a linha vertebral. Na sequência traçou-se uma linha pela extremidade inferior da vértebra caudal da curva; traçou-se, então, um ângulo reto a essa linha. 0 ângulo a ser medido foi o ângulo formado pelas duas linhas perpendiculares às vértebras terminais (20). Todas as medidas foram realizadas pelo mesmo médico ortopedista do Ambulatório de Coluna Vertebral.

\section{Análise estatística}

Os dados foram analisados com o programa estatístico SPSS versão 13.0 (SPSS Inc., Chicago, IL, USA). A análise descritiva foi expressa em média (desvio-padrão) para variáveis numéricas e frequências e porcentagens para variáveis categóricas.

Para estudar a associação entre os domínios dos questionários e da escala EVA com os ângulos de Cobb torácico proximal, torácico principal e lombar, foi utilizado o coeficiente de correlação linear de Pearson (21). Em todas as análises, as correlações foram classificadas da seguinte forma: fraca $(0,3-$ $0,5)$; moderada $(0,5-0,7)$; e forte $(0,7-1)(21)$.

\section{Resultados}

Foi observado na Tabela 1 que 84,2\% dos participantes são do gênero feminino, com uma média de 14,5 anos, $51 \mathrm{~kg}, 1,61 \mathrm{~m}$ de altura e IMC de 18,75 $\mathrm{kg} / \mathrm{m}^{2}$. Os ângulos torácico proximal, torácico principal e lombar apresentaram uma média de $30,1^{\circ}$, $46,8^{\circ}$ e $47^{\circ}$ de Cobb respectivamente.

A Tabela 2 mostra fraca correlação entre os ângulos de Cobb torácico principal e lombar com os domínios do questionário SF-36.

Na Tabela 3, as correlações dos ângulos de Cobb com os domínios do questionário SRS-22 mostraram correlações moderadas entre o ângulo torácico proximal com o domínio da dor; do ângulo torácico principal com os domínios de função, dor, autoimagem, saúde mental; e do ângulo lombar com os domínios de autoimagem e pontuação total. Apenas houve correlação forte entre o ângulo torácico principal com a pontuação total dos domínios.

A Tabela 4 apresenta a correlação dos ângulos de Cobb torácico proximal, torácico principal e lombar com os questionários Oswestry e Roland Morris. Houve correlação moderada do Oswestry com o ângulo proximal, e de ambos os questionários com o 
ângulo lombar. Apresentou-se correlação forte entre o ângulo torácico principal com o Oswestry e Roland Morris.

A Tabela 5 apresenta a correlação dos ângulos torácico proximal, torácico principal e lombar com a escala EVA. Foi observada fraca correlação com o ângulo torácico principal, e moderada correlação com o ângulo lombar.

Tabela 1 - Média e desvio-padrão das características demográficas

\begin{tabular}{lcc}
\hline Características demográficas & Médias & Desvio-padrão \\
\hline Gênero feminino & $84,2 \%$ & - \\
Gênero masculino & $15,8 \%$ & - \\
Idade & 14,5 & $+/-3,1$ \\
Peso $(\mathrm{kg})$ & 51 & $+/-7,9$ \\
Altura $(\mathrm{m})$ & 1,61 & $+/-0,08$ \\
IMC $\left(\mathrm{kg} / \mathrm{m}^{2}\right)$ & 18,75 & $+/-2,7$ \\
Ângulo torácico proximal & $30,1^{\circ}$ & $+/-14$ \\
Ângulo torácico principal & $46,8^{\circ}$ & $+/-17,6$ \\
Ângulo lombar & $47^{\circ}$ & $+/-10,4$ \\
\hline
\end{tabular}

Fonte: Dados da pesquisa.

\section{Discussão}

Foi observada fraca correlação entre os ângulos de Cobb avaliados com os domínios do questionário SF 36. Houve forte correlação entre o ângulo de Cobb torácico principal com a soma total dos domínios do questionário SRS 22, entre os questionários Oswestry e Roland Morris com o ângulo torácico principal e entre o ângulo lombar com a dor.

A qualidade de vida envolve aspectos relacionados à saúde, funcionalidade nas atividades do dia a dia, fatores sociais e psicológicos que são afetados significativamente com a progressão da EIA (9). A abordagem dos questionários SRS-22 e SF-36 é baseada nesses aspectos; contudo, nos indivíduos com EIA existem alterações específicas, decorrentes do desvio lateral, tais como deformidades na caixa torácica, mudanças na configuração corporal e a gibosidade costal, que irão prejudicar a autoimagem e a qualidade de vida desses adolescentes (22).

Como já citado, o questionário SF-36 não especifica sua abordagem com relação às características específicas da EIA. 0 coeficiente de correlação entre os ângulos torácico proximal, torácico principal e lombar com os domínios do SF-36 não mostrou resultado moderado ou forte. De acordo com

Tabela 2 - Correlação dos ângulos de Cobb torácico proximal, torácico principal e lombar com os domínios do questionário SF-36

\begin{tabular}{|c|c|c|c|c|c|c|}
\hline \multirow[b]{2}{*}{ SF-36 (domínios) } & \multicolumn{2}{|c|}{ Cobb torácico proximal } & \multicolumn{2}{|c|}{ Cobb torácico principal } & \multicolumn{2}{|c|}{ Cobb lombar } \\
\hline & $\mathrm{n}$ & $R(p)$ & $\mathrm{n}$ & $R(p)$ & $\mathrm{n}$ & $R(p)$ \\
\hline $\mathrm{CF}$ & 14 & $-0,246(0,397)$ & 12 & $-0,496(0,101)$ & 12 & $-0,433(0,160)$ \\
\hline $\mathrm{AF}$ & 14 & $-0,069(0,814)$ & 12 & $-0,264(0,408)$ & 12 & $-0,439(0,154)$ \\
\hline Dor & 14 & $-0,429(0,126)$ & 12 & $-0,341(0,277)$ & 12 & $-0,340(0,279)$ \\
\hline ES & 14 & $-0,085(0,772)$ & 12 & $-0,096(0,766)$ & 12 & $-0,222(0,488)$ \\
\hline Vit & 14 & $-0,027(0,926)$ & 12 & $-0,396(0,203)$ & 12 & $-0,329(0,296)$ \\
\hline AS & 14 & $-0,163(0,579)$ & 12 & $-0,064(0,843)$ & 12 & $0,260(0,414)$ \\
\hline $\mathrm{AE}$ & 14 & $-0,277(0,338)$ & 12 & $-0,220(0,492)$ & 12 & $0,154(0,632)$ \\
\hline SM & 14 & $-0,428(0,127)$ & 12 & $-0,497(0,100)$ & 12 & $-0,082(0,801)$ \\
\hline
\end{tabular}

Legenda: $\mathrm{CF}=$ capacidade funcional; $\mathrm{AF}=$ aspectos físicos; $\mathrm{ES}=$ estado geral de saúde; Vit $=$ vitalidade; $\mathrm{AS}=$ aspectos sociais; $\mathrm{AE}=$ aspectos emocionais; $S M=$ saúde mental; $n=$ número da amostra; $R=$ coeficiente de correlação de Pearson; $p=$ nível de significância.

Fonte: Dados da pesquisa. 
Ascani et al. (23), 19\% dos indivíduos com EIA possuem alterações psicológicas e, destes, 94\% apresentam curvaturas acima de $40^{\circ}$ de Cobb. Portanto, era esperado que houvesse correlação com valores acima de 0,5 dos ângulos torácico principal e lombar, que obtiveram médias acima de $40^{\circ}$ de Cobb, indicando uma redução da pontuação nos domínios do SF-36 com o aumento dos ângulos. Ou seja, pior qualidade de vida com a severidade da curvatura.

Tabela 3 - Correlação dos ângulos de Cobb torácico proximal, torácico principal e lombar com os domínios do questionário SRS-22

\begin{tabular}{|c|c|c|c|c|c|c|}
\hline \multirow[b]{2}{*}{ SRS-22 (domínios) } & \multicolumn{2}{|c|}{ Cobb torácico proximal } & \multicolumn{2}{|c|}{ Cobb torácico principal } & \multicolumn{2}{|c|}{ Cobb lombar } \\
\hline & $\mathrm{n}$ & $R(p)$ & $\mathrm{n}$ & $R(p)$ & $\mathbf{n}$ & $R(p)$ \\
\hline $\mathrm{FC}$ & 13 & $-0,230(0,450)$ & 13 & $-0,648^{\star}(0,017)$ & 11 & $-0,481(0,134)$ \\
\hline Dor & 13 & $-0,580 *(0,038)$ & 13 & $-0,573^{\star}(0,041)$ & 11 & $0,241(0,475)$ \\
\hline Al & 13 & $-0,304(0,312)$ & 13 & $-0,542^{\star}(0,056)$ & 11 & $-0,619 *(0,042)$ \\
\hline SM & 13 & $-0,194(0,525)$ & 13 & $-0,575^{\star}(0,040)$ & 11 & $-0,200(0,556)$ \\
\hline SF & 13 & $0,348(0,245)$ & 13 & $0,014(0,964)$ & 11 & $-0,199(0,558)$ \\
\hline Total & 13 & $-0,317(0,291)$ & 13 & $-0,706^{\star \star}(0,007)$ & 11 & $-0,580^{*}(0,062)$ \\
\hline
\end{tabular}

Legenda: $\mathrm{FC}=$ função; $\mathrm{Al}=$ autoimagem; $\mathrm{SM}=$ saúde mental; $\mathrm{SF}=$ satisfação com tratamento; $\mathrm{n}=$ número da amostra; $\mathrm{R}=$ coeficiente de correlação de Pearson; * $=$ correlação moderada: $0,5-0,7 ;{ }^{* *}=$ correlação forte: $>0,7 ; p=$ nível de significância.

Fonte: Dados da pesquisa.

Tabela 4 - Correlação dos ângulos de Cobb torácico proximal, torácico principal e lombar com os questionários Oswestry e Roland Morris

\begin{tabular}{|c|c|c|c|c|c|c|}
\hline & \multicolumn{2}{|c|}{ Cobb torácico proximal } & \multicolumn{2}{|c|}{ Cobb torácico principal } & \multicolumn{2}{|c|}{ Cobb lombar } \\
\hline & $n$ & $R(p)$ & $\mathrm{n}$ & $R(p)$ & $\mathbf{n}$ & $R(p)$ \\
\hline Oswestry & 10 & $0,589 *(0,073)$ & 7 & $0,774 * *(0,041)$ & 8 & $0,581 *(0,131)$ \\
\hline Roland Morris & 10 & $0,258(0,472)$ & 8 & $0,725^{\star *}(0,042)$ & 8 & $0,528 *(0,178)$ \\
\hline
\end{tabular}

Legenda: $\mathrm{n}=$ número da amostra; $\mathrm{R}=$ coeficiente de correlação de Pearson; * $=$ correlação moderada: $0,5-0,7 ;{ }^{*}$ * $=$ correlação forte:

$>0,7 ; p=$ nível de significância.

Fonte: Dados da pesquisa.

Tabela 5 - Correlação dos ângulos de Cobb torácico proximal, torácico principal e lombar com a escala de dor EVA

\begin{tabular}{|c|c|c|c|c|c|c|}
\hline \multirow{2}{*}{ EVA } & \multicolumn{2}{|c|}{ Cobb torácico proximal } & \multicolumn{2}{|c|}{ Cobb torácico principal } & \multicolumn{2}{|c|}{ Cobb lombar } \\
\hline & $\mathrm{n}$ & $R(p)$ & $\mathbf{n}$ & $R(p)$ & $\mathbf{n}$ & $R(p)$ \\
\hline & 10 & $0,150(0,680)$ & 8 & $0,385(0,347)$ & 8 & $0,550 *(0,158)$ \\
\hline
\end{tabular}

Legenda: $\mathrm{n}=$ número da amostra; $\mathrm{R}=$ coeficiente de correlação de Pearson; * $=$ correlação moderada: $0,5-0,7 ;{ }^{*}$ * $=$ correlação forte:

$>0,7 ; p=$ nível de significância.

Fonte: Dados da pesquisa. 
Os dados obtidos corroboram com a literatura de que o questionário SF-36 não é discriminativo para avaliar a qualidade de vida na EIA, pois não abrange a complexa interação da qualidade de vida com o grau de deformidade escoliótica $(9,24)$. Além disso, esse questionário não possui domínio sobre a autoimagem, que é uma das alterações mais citadas na escoliose (24).

$\mathrm{Na}$ análise dos ângulos torácico proximal, torácico principal e lombar com o SRS-22 foi mostrada correlação moderada do ângulo torácico principal com os domínios da função, dor, autoimagem, saúde mental; e forte correlação com a pontuação total. De acordo com a análise estatística utilizada, essa correlação foi inversamente proporcional, ou seja, quanto maior o ângulo torácico principal menor a pontuação desses domínios, indicando uma redução na funcionalidade, saúde mental, autoimagem e maior presença de dor nos indivíduos com EIA.

Esses resultados se assemelham aos obtidos no estudo de Asher et al. (11), no qual houve boa correlação da autoimagem, dor, função e saúde mental com o grau da curvatura. Os resultados do presente estudo também corroboram com Aulisa et al. (9), que mostraram que a pontuação do SRS-22 é inversamente proporcional à severidade da curvatura.

Houve correlação moderada do ângulo torácico proximal com o domínio da dor, sendo observado aumento da dor com o aumento do ângulo, e do ângulo lombar com o domínio da autoimagem e pontuação total, tendo, portanto, diminuição da percepção da imagem e na pontuação total com a severidade da curvatura.

A média dos ângulos torácico principal e lombar $\left(46,8^{\circ}\right.$ e $47^{\circ}$, respectivamente) foram acima de $40^{\circ}$ de Cobb, enquanto o ângulo torácico proximal não $\left(30,1^{\circ}\right)$. Esse dado corrobora novamente com Ascani et al. (23), pois na correlação com o SRS-22 o ângulo torácico principal teve cinco domínios com coeficiente de correlação com valores acima de 0,5; o ângulo lombar, dois; e o torácico proximal, apenas um. Portanto, a qualidade de vida mostrou estar mais prejudicada quando os ângulos das curvaturas foram superiores a $40^{\circ}$. De acordo com Parent et al. (7), quando o ângulo de Cobb excede um limite máximo entre $43^{\circ}$ a $48^{\circ}$, os domínios de autoimagem, função, dor e pontuação total do SRS reduzem a cada grau adicionado na curvatura, evidenciando piora na qualidade de vida.

0 estudo de Freidel et al. (25) apresentou resultado controverso, mostrando que a qualidade de vida independe do ângulo da curvatura. Contudo, essa análise foi realizada utilizando o questionário SF-36, que, como dito anteriormente, não faz relação de suas questões com as alterações específicas da escoliose.

Apesar de não ter sido feita, neste estudo, uma correlação da severidade da escoliose com o gênero feminino e masculino, é importante mencionar que a progressão da escoliose e a diminuição da imagem corporal são maiores em adolescentes do gênero feminino que no masculino (26).

Na análise da escala EVA com os ângulos das curvaturas, houve apenas correlação moderada com o ângulo lombar, sendo esta diretamente proporcional, ou seja, quanto maior o ângulo lombar maior foi a intensidade da dor. A incidência de dor nas costas é alta em adolescentes com escoliose quando comparados com a população saudável, e o risco de dor aumenta com a idade (24), sendo vista alta incidência de dor lombar nessa população (24).

Embora a presença de dor nas costas seja frequentemente associada à escoliose, a literatura mostra que a dor na EIA é rara, sendo considerada normalmente indolor, necessitando-se assim da exclusão de patologias de base (27). Segundo a Scoliosis Research Society (4), a dor ocorre em razão da realização de atividades sem a força adequada da musculatura abdominal e dos extensores do tronco, associada à falta de flexibilidade dos músculos isquiotibiais.

A dor lombar é mais comum no gênero feminino, com aumento da idade ou realização de atividades de alto ou baixo nível, e durante o período do estirão do crescimento (28). Em um estudo retrospectivo com 2.442 pacientes com escoliose idiopática e idade entre 6 e 20 anos, foi mostrado que $23 \%$ apresentavam dor na região lombar, e sua prevalência aumentou para $32 \%$ quando avaliados apenas pacientes com idade superior a 15 anos (25). Mesmo com a faixa etária da população deste estudo tendo sido inferior a 15 anos foi mostrada correlação moderada do EVA com o ângulo lombar; contudo, não sendo possível evidenciar correlação dos ângulos torácico proximal e principal com a dor.

Os questionários Roland Morris e Oswestry apresentaram forte coeficiente de correlação com o ângulo torácico principal, e moderado com o ângulo lombar. Esses dados mostram que quanto maior o ângulo maior a pontuação dos questionários e, portanto, maior incapacidade funcional em razão da presença de dor.

O questionário Roland Morris é um dos instrumentos validados mais utilizados para mensurar a 
funcionalidade de atividades do cotidiano que são prejudicadas pela dor lombar $(29,30)$. De acordo com Lauridsen et al. (31), o que difere o Oswestry do Roland Morris é o fato de que o primeiro é mais sensível a alterações de incapacidades elevadas, enquanto o segundo possui maior sensibilidade a alterações de incapacidades de nível mais baixo. As correlações desses questionários com os ângulos das curvaturas foram muito semelhantes, evidenciando que ambos obtiveram a mesma eficácia para avaliar a funcionalidade na EIA.

\section{Conclusão}

Foi constatada forte correlação entre o questionário Oswestry com o ângulo de Cobb torácico principal e entre o questionário Roland Morris com o ângulo de Cobb torácico principal.

\section{Referências}

1. Magee DJ. Coluna torácica (dorsal). Avaliação da postura. In: Magee DJ. Avaliação musculoesquelética. Barueri: Manole; 2005. p. 428-29.

2. Luk KDK, Vidyadhara S. Coupling between sagittal and frontal plane deformity correction in idiopathic thoracic scoliosis and its relationship with postoperative sagittal alignment. Spine. 2010;35(11): 1158-64.

3. Lenke CL. Milwaukee: Scoliosis Research Society. Dedicated to the education, research and treatment of spinal deformity; c1966-2010. 2010 [access 11 set. 2010]. Available at: http://www.srs.org/ professionals/education/adolescent/idiopathic/.

4. Lenke CL. Terminology Committee: SRS terminology committee and working group on spinal classification revised glossary of terms [Internet]. Milwaukee: Scoliosis Research Society - dedicated to the education, research and treatment of spinal deformity; c1966-2010. 2000 [access 20 set. 2010]. Available at: http://www.srs.org/professionals/glossary/glossary.php.

5. Weiss HR, Goodall D. The treatment of adolescent idiopathic scoliosis (AIS) according to present evidence. Eur J Phys Rehabil Med. 2008;44(2):177-93.
6. Busscher I, Wapstra FH, Veldhuizen AG. Predicting growth and curve progression in the individual patient with adolescent idiopathic scoliosis: design of a prospective longitudinal cohort study. BMC Musculoskelet Disord. 2010;11(93):1-9.

7. Parent EC, Wong D, Hill D, Mahood J, Moreau M, Raso J, et al. The association between scoliosis research society-22 scores and scoliosis severity changes at a clinically relevant threshold. Spine. 2010; 35(3):315-22.

8. Weinstein SL, Dolan LA, Cheng JCY, Danielsson A, Morcuende JA. Adolescent idiopathic scoliosis. Lancet. 2008;371(9623):1527-37.

9. Aulisa AG, Guzzanti V, Perisano C, Marzetti E, Specchia A, Giordano M, et al. Determination of quality of life in adolescents with diopathic scoliosis subjected to conservative treatment. Scoliosis. 2010;5(21):1-7.

10. Martinez MC. As relações entre a satisfação com aspectos psicossociais no trabalho e a saúde do trabalhador. [dissertação]. São Paulo: Universidade de São Paulo, Faculdade de Saúde Pública; 2002.

11. Asher MMD, Min Lai S, Burton D, Manna B. Discrimination validity of the scoliosis research society-22 patient questionnaire: relationship to idiopathic scoliosis curve pattern and Curve Size. Spine. 2003; 1(28):74-8.

12. Glowacki MMD, Misterska EMS, Laurentowska M, Mankowski P. Polish adaptation of scoliosis research society-22 Questionnaire. Spine. 2009; 34(10): 1060-5.

13. McHorney CA, Ware JE Jr, Raczek AE. The MOS 36- Item short-form health survey (SF-36): II. Psychometric and clinical tests of validity in measuring physical and mental health constructs. Medical Care. 1993; 31(3):247-63.

14. Ciconelli RM, Ferraz MB, Santos W, Meinão I, Quaresma MR. Tradução para a língua portuguesa e validação do questionário genérico de avaliação de qualidade de vida SF 36. Rev Bras Reumatol. 1999; 39(3):43-50.

15. Haher TR, Gorup JM, Shin TM, Homel P, Merola AA, Grogan DP, et al. Results of the scoliosis research society instrument for evaluation of surgical outcome in adolescent idiopathic scoliosis: a multicenter study of 244 patients. Spine. 1999;24(14):1435-40. 
16. Rosanova GCL, Gabriel BS, Camarini PMF, Gianini PES, Coelho DM, Oliveira AS. Validade concorrente da versão brasileira do SRS-22 com o BR-SF-36. Rev Bras Fisioter. 2010;14(2):121-6.

17. Scott J, Huskisson EC. Graphic representation of pain. Pain. 1976;2(2):175-84.

18. Nusbaum L, Natour J, Ferraz MB, Goldenberg J. Translation, adaptation and validation of the RolandMorris questionnaire - Brazil Roland-Morris. Braz J Med Biol Res. 2001;34(2):203-10.

19. Vigatto R, Alexandre NM, Correa HR Filho. Development of a Brazilian Portuguese version of the Oswestry disability index: cross-cultural adaptation, reliability, and validity. Spine. 2007;32(4):481-6.

20. Lonstein JE. Avaliação do paciente. In: Bradfort DS, Lonstein JE, Moe JH, Ogilvie JW, Winter RB. Escoliose e outras deformidades na coluna: o livro de Moe. São Paulo: Santos; 1994. p. 47-87.

21. Larson R. Análise estatística. In: Larson R, Farber B. Estatística aplicada. São Paulo: Prentice-Hall; 2006. p. 58-71.

22. Rivett LA, Rothberg A, Stewart A, Berkowitz R. The relationship between quality of life and compliance to a brace protocol in adolescents with idiopathic scoliosis: a comparative study. BMC Musculoskelet Disord. 2009;10(5):1-6.

23. Ascani E, Bartolozzi P, Logroscino CA, Marchetti PG, Ponte A, Savini R, et al. Natural history of untreated idiopathic scoliosis after skeletal maturity. Symposium on epidemiology, natural history and non-operative treatment of idiopathic scoliosis. Spine. 1986;11(8):784-89.

24. Tones M, Moss N, Polly DW. A review of quality of life and psychosocial issues in scoliosis. Spine. 2006; 31(26):3027-38.

25. Freidel K, Petermann F, Reichel D, Steiner A, Warschburger P, Weiss HR. Quality of life in women with idiopathic scoliosis. Spine. 2002;27(4):E87-E91.

26. Korovessis P, Zacharatos S, Koureas G, Megas P. Comparative multifactorial analysis of the effects of idiopathic adolescent scoliosis and Scheuermann kyphosis. Eur Spine J. 2007;16(4):537-46.

27. Davies A, Saifuddin A. Imaging of painful scoliosis. Skeletal Radiol. 2009;38(3):207-23.
28. Houghton KM. Review for the generalist: evaluation of low back pain in children and adolescents. Pediatri Rheumatol. 2010;8:28.

29. Pellisé F, Balagué F, Rajmil L, Cedraschi C, Aguirre M, Fontecha CG, et al. Prevalence of low back pain and its effect on health-related quality of life in adolescents. Arch Pediatr Adolesc Med. 2009;163(1):65-71.

30. Aquino CF, Cardoso VA, Machado NC, Franklin JS, Augusto VG. Análise da relação entre dor lombar e desequilíbrio de força muscular em bailarinas. Fisioter Mov. 2010;23(3):399-408.

31. Lauridsen HH, Hartvigsen J, Manniche C, Korsholm L, Grunnet-Nilsson N. Responsiveness and minimal clinically important difference for pain and disability instruments in low back pain patients. BMC Musculoskelet Disord. 2006;7(82):1-16.

Recebido: 20/12/2010

Received: $12 / 20 / 2010$

Aprovado: 30/05/2011

Approved: 05/30/2011 